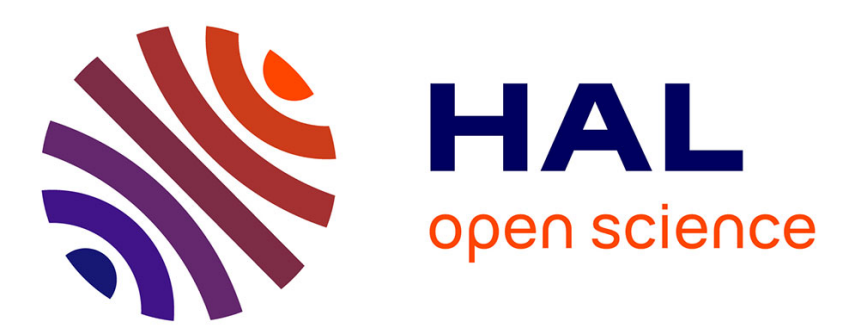

\title{
A phase-field model for brittle fracture of anisotropic materials
}

\author{
Hela Gmati, Charles Mareau, Amine Ammar, Saber El Arem
}

\section{To cite this version:}

Hela Gmati, Charles Mareau, Amine Ammar, Saber El Arem. A phase-field model for brittle fracture of anisotropic materials. International Journal for Numerical Methods in Engineering, 2020, 121 (15), pp.3362-3381. 10.1002/nme.6361 . hal-02944667

\section{HAL Id: hal-02944667 https://hal.science/hal-02944667}

Submitted on 21 Sep 2020

HAL is a multi-disciplinary open access archive for the deposit and dissemination of scientific research documents, whether they are published or not. The documents may come from teaching and research institutions in France or abroad, or from public or private research centers.
L'archive ouverte pluridisciplinaire HAL, est destinée au dépôt et à la diffusion de documents scientifiques de niveau recherche, publiés ou non, émanant des établissements d'enseignement et de recherche français ou étrangers, des laboratoires publics ou privés. 


\section{A phase-field model for brittle fracture of anisotropic materials}

\section{Hela Gmati@ | Charles Mareau | Amine Ammar $『$ | Saber El Arem}

LAMPA, Arts et Métiers ParisTech, Angers, France

\section{Correspondence}

Hela Gmati, LAMPA, Arts et Métiers ParisTech, 2 Boulevard du Ronceray, BP 93525, F-49035 Angers Cedex 01, France. Email: hela.gmati@ensam.eu

\begin{abstract}
Summary
In the present work, a phase field damage model is developed to address the numerical simulation of brittle fracture. This model successfully captures some important aspects of crack propagation, including crack branching and bifurcation. In addition, the proposed phase field model has been developed in the general framework of anisotropic elasticity. It can thus be used for the simulation of brittle fracture in polycrystalline materials, for which crack propagation is impacted by crystallographic orientation because of the anisotropic character of stiffness properties.
\end{abstract}

\section{K E Y W O R D S}

anisotropic elasticity, crack propagation, damage-gradient model, finite elements, phase field method

\section{1 | INTRODUCTION}

The prediction of failure due to crack initiation and propagation through computational models is an important challenge in materials science and solid state physics. The theoretical foundation of brittle fracture uses the work of Griffith, ${ }^{1}$ which considers the competition between elastic strain energy and surface energy to determine whether the conditions for fracture are met or not. However, because the approach of Griffith is inappropriate for dealing with phenomena such as nucleation or branching, some alternative methods have been proposed. Specifically, in the recent years, the Phase Field Method (PFM) has emerged as an attractive approach for the description of brittle fracture. ${ }^{2-5}$ Generally speaking, the PFM is appropriate for dealing with moving boundary problems (eg, phase transitions). The PFM relies on the introduction of a field variable, which plays the role of an order parameter, to obtain a smooth description of the interfaces in multiphase systems. In the context of fracture, the PFM uses the ingredients of continuum damage mechanics ${ }^{6}$ to treat the order parameter as a damage variable representing the degradation of mechanical properties. The PFM considers the damage variable and its spatial gradient as state variables to obtain a smooth approximation of cracked surfaces. Phase field models of fracture can thus be interpreted as damage gradient models ${ }^{7,8}$ for which the free energy includes a damage gradient term associated with a regularizing internal length scale. The PFM allows dealing with complex fracture problems and stands as an interesting alternative to element enrichment strategies, ${ }^{9}$ nodal enrichment strategies, ${ }^{10}$ or thick level-set methods. ${ }^{11}$ For instance, the PFM has been used by Nguyen et $\mathrm{al}^{12}$ to model the behavior of cementitious materials. The works of Larsen, ${ }^{13}$ Bourdin et al, ${ }^{14}$ Borden et al, ${ }^{15}$ and Hofacker and Miehe ${ }^{5}$ have shown that the PFM can be extended to dynamic fracture and produce results that agree properly with experimental observations. The works of References 16 and 17 have shown that the PFM can deal with anisotropic fracture. These works are mainly focused on the anisotropy of fracture energy only and they do not include anisotropy related to the properties of the materials. Indeed, anisotropy is included through a fourth-order tensor penalizing fracture interfaces in different directions and by including higher order gradient term in the crack energy density function. 
An important aspect for phase field models of brittle fracture is their ability to correctly reproduce the impact of loading conditions. Specifically, to describe the asymmetric behavior of brittle materials (ie, tension/compression asymmetry), different strategies have been employed. The phase field model of Reference 3 uses the decomposition of the strain tensor into positive and negative parts to include tension/compression asymmetry. An alternative option, which has been proposed by Amor et al, ${ }^{18}$ is based on the separation of the strain tensor into spherical and deviatoric contributions. Closure effects are then handled by considering the impact of damage on stiffness properties differently depending on the sign of the spherical strain tensor. These strategies have been compared to each other by Ambati et al, ${ }^{19}$ who also proposed a hybrid formulation to reduce the computational cost of finite element simulations of brittle fracture with the PFM. Recently, another alternative, which considers the crack's orientation to define the driving stress and the corresponding driving strain potential, has been introduced by Steinke et al. ${ }^{20}$ They postulate a directional split analysis of the crack closure behaviour of isotropic linear elastic material, which overcomes some issues of the previous volumetric-deviatoric and spectral splits.

In this work, a nonlocal damage model, which considers a scalar damage variable and its gradient as state variables, is constructed. Following the suggestion of Reference 18, the consideration of closure effects relies on the separation of the elastic strain energy into spherical and deviatoric contributions. However, while the original proposition of Reference 18 is restricted to isotropic elasticity, the proposed model is adapted to any class of material symmetry. Also, particular care is taken to introduce some material parameters to control the respective impacts of the spherical and deviatoric contributions on the development of damage. This article is organized as follows. In Section 2, the diffuse approximation of fracture surfaces is briefly recalled. The constitutive relations are detailed in Sections 3 and 4 . The numerical method used for the resolution of equilibrium and compatibility equations is described in Section 5. Finally, in Section 6, some numerical examples are presented.

\section{2 | CRACK DENSITY FUNCTIONAL}

In engineering materials, the degradation of mechanical properties results from the creation and the propagation of internal discontinuity surfaces known as cracks. For the evaluation of the total cracked surface area $S_{\mathrm{c}}$ in a body $B$, the PFM relies on the introduction of a damage phase field $d$. For a material point, whose position at time $t$ is denoted by $\boldsymbol{x}$, the value of the phase field $d(\boldsymbol{x}, t)$ is comprised between zero and unity*. The unity value corresponds to a fully damaged state while an undamaged material point is given a zero value. The evaluation of the total cracked surface area $S_{\mathrm{c}}$ requires the introduction of a crack density functional $\gamma$ which depends on both the phase field $d$ and its gradient $\boldsymbol{\nabla} d$ :

$$
S_{\mathrm{c}}=\int_{\Omega} \gamma(d, \nabla d) d V
$$

where $\Gamma$ is the boundary of $\Omega$ and $\Omega$ is the region in space that is occupied by the body $\mathcal{B}$ at time $t$. Different propositions ${ }^{21-23}$ have been made for the construction of the crack density functional $\gamma$. The functional proposed by Pham et al, ${ }^{22}$ which has been used by Bleyer et al, ${ }^{24}$ is adopted in the present work. This functional takes the following form:

$$
\gamma(d, \nabla d)=\frac{3}{8 l_{\mathrm{c}}}\left(d+l_{\mathrm{c}}^{2} \nabla d \cdot \nabla d\right) .
$$

In the above expression, $l_{\mathrm{c}}$ is an internal length controlling the crack thickness, the discrete description being retrieved when $l_{\mathrm{c}}$ approaches zero. The above definition of the crack density functional $\gamma$ is such that the surface energy obtained for the localized 1D-solution of a bar in traction will be precisely equal to $g_{c}$, identified to the fracture energy of the Griffith model.

\section{3 | BALANCE EQUATIONS}

For an isothermal process, the response of the body $\mathcal{B}$ is given by the evolution of the displacement field $\boldsymbol{u}$ and the phase field $d$, which represents the progression of damage. The evolution of these fields is governed by some equilibrium

*The present work is based on the infinitesimal deformation theory. No distinction between the initial and current positions of a material point is therefore made. 
equations which are commonly derived from the application of an extended principle of virtual power. ${ }^{25}$ More specifically, in a Galilean frame, for any admissible virtual motion $\left\{\dot{\boldsymbol{u}}^{\star}, \dot{d}^{\star}\right\}$, the power developed by external forces $\mathcal{P}^{\mathrm{e}}$ is equal to the sum of the change in total kinetic energy $\dot{\mathcal{K}}$ and the power developed by internal forces $\mathcal{P}^{\mathrm{i}}$ :

$$
\mathcal{P}^{\mathrm{e}}=\dot{\mathcal{K}}+\mathcal{P}^{\mathrm{i}}
$$

The external forces contributing to $\mathcal{P}^{\mathrm{e}}$ are represented by a body force density $\boldsymbol{b}$ and a contact force density $\boldsymbol{t}$. The power of external forces is thus given by:

$$
\mathcal{P}^{\mathrm{e}}=\int_{\Omega} \boldsymbol{b} \cdot \dot{\boldsymbol{u}}^{\star} d V+\int_{\partial \Omega} \boldsymbol{t} \cdot \dot{\boldsymbol{u}}^{\star} d S
$$

Following the terminology of Reference 26, the above expression for the power developed by external forces corresponds to an insulation condition in the sense that no external force is associated with the damage phase field.

In contrast with the classical formulation of continuum mechanics, the power of internal forces $\mathcal{P}^{\mathrm{i}}$ includes the additional contributions of $\dot{d}^{\star}$ and $\boldsymbol{\nabla} \dot{d}^{\star}$ so that:

$$
\mathcal{P}^{\mathrm{i}}=\int_{\Omega} \boldsymbol{\sigma}: \nabla \dot{\boldsymbol{u}}^{\star} d V+\int_{\Omega} \xi \dot{d}^{\star} d V+\int_{\Omega} \boldsymbol{\eta} \cdot \nabla \dot{d}^{\star} d V
$$

where $\sigma$ is the stress tensor, $\xi$ is the force associated with the damage variable $d$, and $\boldsymbol{\eta}$ is the force associated with its spatial gradient $\boldsymbol{\nabla} d$. The rate of change in kinetic energy $\dot{\mathcal{K}}$ is:

$$
\dot{\mathcal{K}}=\int_{\Omega} \rho \ddot{\boldsymbol{u}} \cdot \dot{\boldsymbol{u}}^{\star} d V
$$

where $\rho$ is the mass density.

The principle of virtual power, whose statement is provided by Equation (3), is satisfied for any $\left\{\dot{\boldsymbol{u}}^{\star}, \dot{d}^{\star}\right\}$ if the stress field $\sigma$ is such that:

$$
\begin{gathered}
\operatorname{div} \boldsymbol{\sigma}+\boldsymbol{b}=\rho \ddot{\boldsymbol{u}} \quad \forall \boldsymbol{x} \text { in } \Omega \\
\boldsymbol{\sigma} \cdot \boldsymbol{n}=\boldsymbol{t} \quad \forall \boldsymbol{x} \text { in } \partial \Omega .
\end{gathered}
$$

In a similar fashion, the fields $\xi$ and $\eta$ must meet with the following conditions:

$$
\begin{gathered}
\operatorname{div} \boldsymbol{\eta}-\xi=0 \quad \forall \boldsymbol{x} \text { in } \Omega \\
\boldsymbol{\eta} \cdot \boldsymbol{n}=0 \quad \forall \boldsymbol{x} \text { in } \partial \Omega .
\end{gathered}
$$

The evolution of the body $\mathcal{B}$, when subjected to the boundary conditions given by (8) and (10), is governed by the equilibrium equations (7) and (9).

\section{4 | CONSTITUTIVE EQUATIONS}

In order to model the evolution of the body $\mathcal{B}$, the equilibrium equations must be supplemented with some constitutive equations. The state variables used for the construction of constitutive equations are the strain tensor $\varepsilon$, the damage variable $d$, and its gradient $\boldsymbol{\nabla} d$. Because no additional internal variable (eg, plastic strain tensor, hardening variable) is introduced, the behavior of a material point is assumed to be purely elastic. In the following, the constitutive equations are developed within the framework of generalized standard materials. ${ }^{27}$ Within this framework, constitutive relations are obtained from a state potential $\psi$ and a dissipation potential $\phi$ which are such that:

$$
\sigma=-\partial_{\varepsilon} \psi
$$




$$
\begin{gathered}
\xi=\partial_{d} \psi+\partial_{\dot{d}} \phi \\
\boldsymbol{\eta}=\partial_{\nabla d} \psi
\end{gathered}
$$

The state potential $\psi$, which corresponds to the free energy density, is decomposed into volume and surface contributions denoted, respectively, $\psi_{\mathrm{v}}$ and $\psi_{\mathrm{s}}$ :

$$
\psi(\varepsilon, d, \nabla d)=\psi_{\mathrm{v}}(\varepsilon, d)+\psi_{\mathrm{s}}(d, \nabla d) .
$$

The surface contribution $\psi_{\mathrm{s}}$ uses the crack density $\gamma$, which is given by Equation (2), and the surface energy density $g_{\mathrm{c}}$ :

$$
\begin{aligned}
& \psi_{\mathrm{s}}(d, \nabla d)=g_{\mathrm{c}} \gamma(d, \nabla d) \\
& =\frac{3 g_{\mathrm{c}}}{8 l_{\mathrm{c}}}\left(d+l_{\mathrm{c}}^{2} \nabla d \cdot \nabla d\right) .
\end{aligned}
$$

The volume contribution $\psi_{\mathrm{v}}$ depends on the stress tensor $\varepsilon$ according to:

$$
\psi_{\mathrm{v}}(\varepsilon, d)=\frac{1}{2} \varepsilon: \mathbb{C}: \varepsilon
$$

The stiffness tensor $\mathbb{C}$ depends on both the damage variable $d$ and the strain tensor $\varepsilon$. Specifically, to account for closure effects, two different situations, depending on whether the volume increases or decreases, are considered:

$$
\mathbb{C}= \begin{cases}\mathbb{C}_{t} & \text { if } \operatorname{tr}(\varepsilon) \geq 0 \\ \mathbb{C}_{\mathrm{c}}=\mathbb{C}_{\mathrm{t}}+\mathbb{P}_{\mathrm{s}}:\left(\tilde{\mathbb{C}}-\mathbb{C}_{\mathrm{t}}\right): \mathbb{P}_{\mathrm{s}} & \text { if } \operatorname{tr}(\varepsilon)<0\end{cases}
$$

with $^{\dagger}$ :

$$
\mathbb{C}_{\mathrm{t}}=\left(\tilde{\mathbb{C}}^{-1}+g(d)\left(f_{\mathrm{s}} \mathbb{P}_{\mathrm{s}}: \tilde{\mathbb{C}}^{-1}: \mathbb{P}_{\mathrm{s}}+f_{\mathrm{d}} \mathbb{P}_{\mathrm{d}}: \tilde{\mathbb{C}}^{-1}: \mathbb{P}_{\mathrm{d}}\right)\right)^{-1}
$$

In the above equations, $\tilde{\mathbb{C}}$ denotes the initial (ie, undamaged) elastic stiffness tensor. Also, according to Equation (18), the spherical part of stiffness properties is recovered for negative volume changes. The definition of the stiffness tensor therefore uses the spherical and deviatoric projection tensors, $\mathbb{P}_{\mathrm{s}}$ and $\mathbb{P}_{\mathrm{d}}$, which are given by:

$$
\begin{gathered}
\mathbb{P}_{\mathrm{s}}=\frac{1}{3}(\boldsymbol{I} \otimes \boldsymbol{I}) \\
\mathbb{P}_{\mathrm{d}}=\mathbb{I}-\frac{1}{3}(\boldsymbol{I} \otimes \boldsymbol{I}) .
\end{gathered}
$$

The material parameters $f_{\mathrm{s}}$ and $f_{\mathrm{d}}$, which have been introduced in Equation 18, allow controlling the contributions of spherical and deviatoric strains to the development of damage. The degradation function $g(d)$ displays the following properties:

$$
\begin{gathered}
g(0)=0 \\
g(1)=\infty \\
g^{\prime}(d) \geq 0, \quad \forall d \text { in }[0,1] .
\end{gathered}
$$

Condition (22) is needed for stiffness properties to be unaltered in the absence of damage. Also, according to condition (23), the stiffness properties of a fully damaged material point completely vanishes when $\operatorname{tr}(\varepsilon) \geq 0$. Finally, when condition

\footnotetext{
${ }^{\dagger}$ For generalization purpose, one could include some cross-terms in the definition of $\mathbb{C}_{t}$ to consider interactions between deviatoric and spherical strains. These terms, which are not considered here for simplicity, necessarily vanish for isotropic and cubic materials.
} 
(24) is fulfilled, for a fixed strain state, the progression of damage always results in a decrease of stiffness properties. In the present work, the degradation function $g(d)$ has the following form:

$$
g(d)=\frac{d}{1-d} .
$$

The constitutive equation for $\boldsymbol{\eta}$, which is the dual variable of $\boldsymbol{\nabla} d$, is obtained from (13):

$$
\eta=\frac{3 g_{\mathrm{c}} l_{\mathrm{c}}}{4} \nabla d
$$

To obtain the evolution rule for the damage variable, the expression of the dissipation potential $\phi$ has to be established. In the present work, a purely local formulation is adopted since the dissipation potential depends solely on the damage variable $d$ and its evolution rate $\dot{d}$ :

$$
\phi(\dot{d}, d)= \begin{cases}\frac{K}{N+1} \frac{\dot{d}^{N+1}}{(1-d)}, & \dot{d} \geq 0 \\ \infty, & \dot{d}<0\end{cases}
$$

where $K$ and $N$ are viscosity parameters (with $K>0$ and $N \geq 0$ ). The parameter $N$ controls the rate sensitivity of damage development. The specific case of a rate insensitive behavior corresponds to $N=0$, in which case the dissipation potential is linearly homogeneous with respect to $\dot{d}$.

Though $\phi$ is not differentiable with respect to $\dot{d}$ around $\dot{d}=0$, the convexity of the dissipation potential allows calculating the subdifferential $\partial_{\dot{d}} \phi$ which is such that:

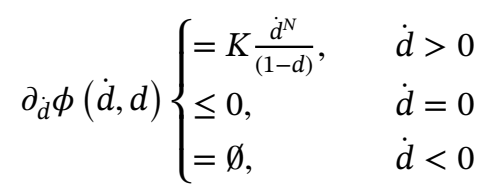

The application of (12) leads to the expression of the driving force for damage $\xi$ :

$$
\xi=\tau+\frac{3 g_{\mathrm{c}}}{8 l_{\mathrm{c}}}+\partial_{\dot{d}} \phi(\dot{d}, d)
$$

where $\tau$ denotes the elastic energy restitution rate:

$$
\tau=\frac{1}{2} \varepsilon: \partial_{d} \mathbb{C}: \varepsilon
$$

with:

$$
\begin{gathered}
\partial_{\mathrm{d}} \mathbb{C}= \begin{cases}\partial_{\mathrm{d}} \mathbb{C}_{\mathrm{t}} & \text { if } \operatorname{tr}(\varepsilon) \geq 0 \\
\partial_{\mathrm{d}} \mathbb{C}_{\mathrm{c}}=\partial_{\mathrm{d}} \mathbb{C}_{\mathrm{t}}-\mathbb{P}_{\mathrm{s}}: \partial_{\mathrm{d}} \mathbb{C}_{\mathrm{t}}: \mathbb{P}_{\mathrm{s}} & \text { if } \operatorname{tr}(\varepsilon)<0\end{cases} \\
\partial_{\mathrm{d}} \mathbb{C}_{\mathrm{t}}=-g^{\prime}(d) \mathbb{C}_{\mathrm{t}}:\left(f_{\mathrm{s}} \mathbb{P}_{\mathrm{s}}: \tilde{\mathbb{C}}^{-1}: \mathbb{P}_{\mathrm{s}}+f_{\mathrm{d}} \mathbb{P}_{\mathrm{d}}: \tilde{\mathbb{C}}^{-1}: \mathbb{P}_{\mathrm{d}}\right): \mathbb{C}_{\mathrm{t}} .
\end{gathered}
$$

Combining the above relations together with the balance equation (9), one obtains the evolution rule for the damage variable:

$$
K \dot{d}^{N}=\left\langle-\tau+\frac{3 g_{\mathrm{c}} l_{\mathrm{c}}}{4} \Delta d-\frac{3 g_{\mathrm{c}}}{8 l_{\mathrm{c}}}\right\rangle(1-d)
$$

According to the evolution equation of the damage variable, damage healing is not allowed (ie, $\dot{d} \geq 0$ ) and the damage variable cannot exceed unity (ie, $\dot{d}=0$ for $d=1$ ). 
As will be discussed in the upcoming result section, the proposed constitutive model offers two advantages. First, closure effects, which favors the development of damage in tension, are accounted for and can be controlled with the $f_{\mathrm{s}}$ and $f_{\mathrm{d}}$ parameters. Second, the above constitutive relations do not require any assumption regarding material symmetry.

\section{5 | NUMERICAL IMPLEMENTATION}

In the present work, the finite element method is used for the resolution of field equations. The numerical implementation of this method for the specific case of the proposed model is briefly discussed here.

\section{1 | Displacement field}

The evolution of the displacement field $\boldsymbol{u}$ is governed by the following field equations:

$$
\begin{gathered}
\operatorname{div} \boldsymbol{\sigma}=\rho \ddot{\boldsymbol{u}} \\
\sigma=\mathbb{C}(d): \varepsilon \\
\varepsilon=\operatorname{sym}(\boldsymbol{\nabla} \boldsymbol{u}) .
\end{gathered}
$$

In the above set of equations, which result from equilibrium and compatibility conditions as well as from constitutive assumptions, the effect of external volume forces has been excluded. The corresponding variational formulation of this problem is given by:

$$
\int_{\Omega} \boldsymbol{u}^{\star} \cdot \operatorname{div} \boldsymbol{\sigma} \mathrm{d} V=\int_{\Omega} \rho \boldsymbol{u}^{\star} \cdot \ddot{\boldsymbol{u}} \mathrm{d} V
$$

where $\boldsymbol{u}^{\star}$ is the test displacement field. After integration by parts, one obtains:

$$
\int_{\Omega} \rho \boldsymbol{u}^{\star} \cdot \ddot{\boldsymbol{u}} \mathrm{d} V+\int_{\Omega} \varepsilon^{\star}: \sigma \mathrm{d} V=0
$$

where $\varepsilon^{\star}$ is the test strain field derived from $\boldsymbol{u}^{\star}$.

Within the context of the finite element method, the introduction of interpolation functions $\boldsymbol{N}$ allows expressing the displacement fields $\boldsymbol{u}$ and $\boldsymbol{u}^{\star}$ from the nodal displacement vectors $\boldsymbol{U}$ and $\boldsymbol{U}^{\star}$ as follows:

$$
\begin{gathered}
\boldsymbol{u}(\boldsymbol{x}, t)=\boldsymbol{N}(\boldsymbol{x}) \boldsymbol{U}(t) \\
\boldsymbol{u}^{\star}(\boldsymbol{x}, t)=\boldsymbol{U}^{\star^{T}}(t) \boldsymbol{N}^{T}(\boldsymbol{x}) .
\end{gathered}
$$

Using the interpolation functions $\boldsymbol{N}$, the first term in (38) becomes:

$$
\int_{\Omega} \rho \boldsymbol{u}^{\star} \cdot \ddot{\boldsymbol{u}} \mathrm{d} V=\boldsymbol{U}^{\star^{T}}\left(\int_{\Omega} \rho \boldsymbol{N}^{T} \cdot \boldsymbol{N} \mathrm{d} V\right) \ddot{\boldsymbol{U}}=\boldsymbol{U}^{\star^{T}} \boldsymbol{M} \ddot{\boldsymbol{U}}
$$

where $\boldsymbol{M}$ is the mass matrix. Also, the strain fields $\varepsilon$ and $\varepsilon^{\star}$ are obtained from $\boldsymbol{U}$ and $\boldsymbol{U}^{\star}$ with:

$$
\begin{gathered}
\boldsymbol{\varepsilon}(\boldsymbol{x}, t)=\boldsymbol{B}(\boldsymbol{x}) \boldsymbol{U}(t) \\
\boldsymbol{\varepsilon}^{\star}(\boldsymbol{x}, t)=\boldsymbol{U}^{\star^{T}}(t) \boldsymbol{B}^{T}(\boldsymbol{x}) .
\end{gathered}
$$


The matrix $\boldsymbol{B}(\boldsymbol{x})$ is defined by the first derivative of the shape functions. In the 2D case, one finds that:

$$
\boldsymbol{B}(\boldsymbol{x})=\left(\begin{array}{ccccc}
\frac{\partial N_{1}(\boldsymbol{x})}{\partial x} & 0 & \ldots & \frac{\partial N_{n}(x)}{\partial x} & 0 \\
0 & \frac{\partial N_{1}(x)}{\partial y} & \cdots & 0 & \frac{\partial N_{n}(x)}{\partial y} \\
\frac{\partial N_{1}(x)}{\partial x} & \frac{\partial N_{1}(x)}{\partial y} & \cdots & \frac{\partial N_{n}(x)}{\partial x} & \frac{\partial N_{n}(x)}{\partial y}
\end{array}\right) .
$$

The second term in Equation (38) is thus given by:

$$
\int_{\Omega} \varepsilon^{\star}: \sigma \mathrm{d} V=\boldsymbol{U}^{\star^{T}}\left(\int_{\Omega} \boldsymbol{B}^{T}: \mathbb{C}: \boldsymbol{B} \mathrm{d} V\right) \boldsymbol{U}=\boldsymbol{U}^{\star^{T}} \boldsymbol{K} \boldsymbol{U}
$$

where $\boldsymbol{K}$ is the stiffness matrix of the system.

The solution of the displacement problem consists in determining the nodal displacement vector $\boldsymbol{U}$. After elimination of the test displacement field from the variational formulation, the nodal acceleration vector $\ddot{U}$ is obtained from the resolution of the following system:

$$
\boldsymbol{M} \ddot{\boldsymbol{U}}(t)+\boldsymbol{K} \boldsymbol{U}(t)=\mathbf{0}
$$

In the present work, $\boldsymbol{U}$ is determined from $\ddot{\boldsymbol{U}}$ with the Newmark method :

$$
\begin{gathered}
\boldsymbol{U}(t+\Delta t)=\boldsymbol{U}(t)+\Delta t \dot{\boldsymbol{U}}(t)+\frac{\Delta t^{2}}{2}((1-b) \ddot{\boldsymbol{U}}(t)+b \ddot{\boldsymbol{U}}(t+\Delta t)) \\
\dot{\boldsymbol{U}}(t+\Delta t)=\dot{\boldsymbol{U}}(t)+\Delta t((1-a) \ddot{\boldsymbol{U}}(t)+a \ddot{\boldsymbol{U}}(t+\Delta t))
\end{gathered}
$$

where $\Delta t$ is the time step while, $a$ and $b$ are time integration parameters.

\section{2 | Damage field}

For the evolution of the damage field $d$, a discrete form of the following nonlocal equation is first needed:

$$
K \dot{d}^{N}=\left(\left\langle-\tau+\frac{3 g_{\mathrm{c}} l_{\mathrm{c}}}{4} \Delta d-\frac{3 g_{\mathrm{c}}}{8 l_{\mathrm{c}}}\right\rangle(1-d)\right) .
$$

To circumvent the difficulty related to the impossibility for damage healing to occur (ie, $\dot{d} \geq 0$ ) and the presence of the power $N$, a local (ie, point-by-point) resolution strategy is adopted here. The sole obstacle for this strategy is the presence of the nonlocal Laplacian diffusion operator, which can be overcome with the construction of a local Laplacian diffusion operator obtained from the resolution of the problem $\theta=\Delta d$ in a weak form. For this purpose, the following nonlocal equation is considered:

$$
\int_{\Omega} d^{\star} \theta \mathrm{d} V=\int_{\Omega} d^{\star} \Delta d \mathrm{~d} V
$$

where $d^{\star}$ is the test damage field. Integrating by parts and using the divergence theorem, one obtains:

$$
\int_{\Omega} d^{\star} \theta d V=\int_{\partial \Omega} d^{\star}(\nabla d \cdot \boldsymbol{n}) d S-\int_{\Omega} \nabla d^{\star} \cdot \nabla d d V
$$

Combining the boundary condition (10) with the constitutive relation (26), the surface integral in the above equation vanishes so that:

$$
\int_{\Omega} d^{\star} \theta d V+\int_{\Omega} \nabla d^{\star} \cdot \nabla d d V=0
$$


Within the context of the finite element method, the damage variables $d$ and $d^{\star}$ at position $\boldsymbol{x}$ are evaluated from the interpolation function $\boldsymbol{N}$ and the nodal damage vectors $\boldsymbol{D}$ and $\boldsymbol{D}^{\star}$ with:

$$
\begin{gathered}
d(\boldsymbol{x}, t)=\boldsymbol{N}(\boldsymbol{x}) \boldsymbol{D}(t) \\
d^{\star}(\boldsymbol{x}, t)=\boldsymbol{D}^{\star^{T}}(t) \boldsymbol{N}^{T}(\boldsymbol{x}) .
\end{gathered}
$$

In a similar fashion, the spatial gradients of the damage variables $\boldsymbol{\nabla} d$ and $\boldsymbol{\nabla} d^{\star}$ are obtained from $\boldsymbol{D}$ and $\boldsymbol{D}^{\star}$ according to:

$$
\begin{gathered}
\boldsymbol{\nabla} d(\boldsymbol{x}, t)=\boldsymbol{Q}(\boldsymbol{x}) \boldsymbol{D}(t) \\
\boldsymbol{\nabla} d^{\star}(\boldsymbol{x}, t)=\boldsymbol{D}^{\star^{T}}(t) \boldsymbol{Q}^{T}(\boldsymbol{x}) \\
\boldsymbol{Q}(\boldsymbol{x})=\left(\begin{array}{ccc}
\frac{\partial N_{1}(x)}{\partial x} & \ldots & \frac{\partial N_{n}(x)}{\partial x} \\
\frac{\partial N_{1}(x)}{\partial y} & \ldots & \frac{\partial N_{n}(x)}{\partial y} .
\end{array}\right)
\end{gathered}
$$

The vector $\Theta$, which contains the nodal values of the Laplacian term $\theta$, can therefore be determined from the solution of:

$$
\boldsymbol{A} \boldsymbol{\Theta}(t)+\boldsymbol{Z} \boldsymbol{D}(t)=\mathbf{0}
$$

with:

$$
\begin{gathered}
\boldsymbol{A}=\int_{\Omega} \boldsymbol{N}^{T} \boldsymbol{N} d V \\
\boldsymbol{Z}=\int_{\Omega} \boldsymbol{Q}^{T} \cdot \boldsymbol{Q} d V,
\end{gathered}
$$

where $\boldsymbol{A}$ is the lumped mass matrix.

Once the vector $\boldsymbol{\Theta}=-\boldsymbol{A}^{-1} \boldsymbol{Z D}$ is known, the damage rate vector $\dot{\boldsymbol{D}}$ is obtained from:

$$
K \dot{\boldsymbol{D}}^{\circ N}(t)=\left\langle-\boldsymbol{\Upsilon}(t)+\frac{3 g_{\mathrm{c}} l_{\mathrm{c}}}{4} \boldsymbol{\Theta}(t)-\frac{3 g_{\mathrm{c}}}{8 l_{\mathrm{c}}} \boldsymbol{D}(t)\right\rangle \circ(\mathbf{1}-\boldsymbol{D}(t))
$$

where $\circ$ is the symbol of Hadamard product, $\mathbf{1}$ is the vector containing the value of 1 in each component and $\mathbf{\Upsilon}(t)$ is the vector containing the nodal values of the elastic energy restitution rate $\tau$. In order to obtain the nodal values straight from the integration point values, a mapping projector is used. For time integration of nodal damage variables, an explicit time integration scheme is employed for the temporal discretization. The Euler explicit method is applied to the time integration of the damage vector:

$$
\boldsymbol{D}(t+\Delta t)=\boldsymbol{D}(t)+\dot{\boldsymbol{D}}(t) \Delta t
$$

\section{3 | Overall algorithm}

The overall algorithm for the estimation of the displacement and damage fields is briefly described here. It is composed of the following steps:

\section{- Initialization}

1. Initialize the nodal displacement vector $\boldsymbol{U}\left(t_{0}\right)$, the nodal velocity vector $\dot{\boldsymbol{U}}\left(t_{0}\right)$ and the nodal damage vector $\boldsymbol{D}\left(t_{0}\right)$.

2. Compute the matrices $\boldsymbol{A}$ and $\boldsymbol{Z}$. 
F I G U R E 1 Single-edge-notched tensile test: boundary conditions, specimen geometry, and mesh [Colour figure can be viewed at wileyonlinelibrary.com]
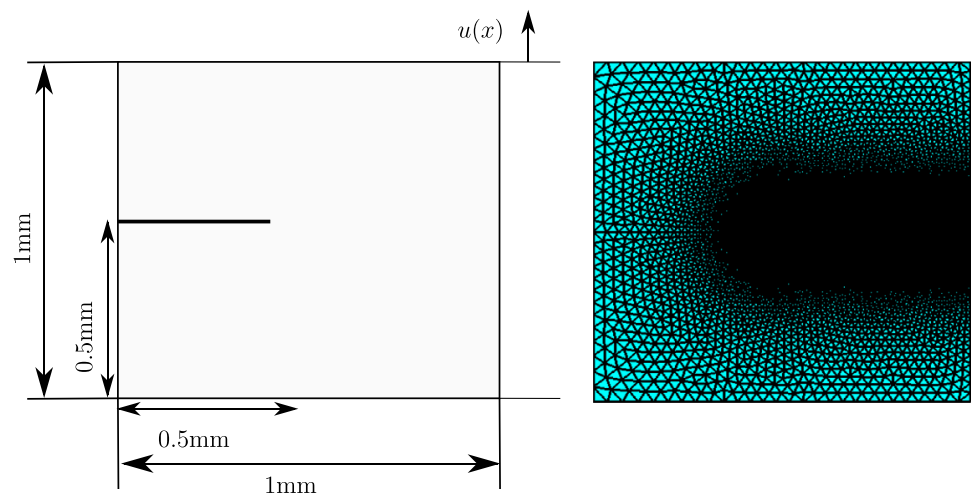

\section{- For each time $t$}

1. For each integration point, compute the strain tensor $\varepsilon(t)$ and the damage variable $d(t)$.

2. For each integration point, compute the stress tensor $\sigma(t)$ and the stiffness tensor $\mathbb{C}(t)$.

3. Compute the stiffness matrix $\boldsymbol{K}(d)$ and the mass matrix $\boldsymbol{M}$ of the system.

4. Compute the nodal acceleration vector $\ddot{\boldsymbol{U}}(t)$.

5. Compute the vector $\Theta(t)$ containing the nodal values of $\theta(t)=\Delta d(t)$.

6. For each integration point, compute the elastic energy restitution rate $\tau(t)$.

7. Compute the vector $\mathbf{Y}(t)$ containing the nodal values of $\tau(t)$.

8. Compute the nodal damage rate vector $\dot{\boldsymbol{D}}(t)$.

9. Update the nodal displacement vector $\boldsymbol{U}(t+\Delta t)$ and the nodal damage vector $\boldsymbol{D}(t+\Delta t)$.

\section{- End}

The present code has been implemented in Matlab.

\section{6 | NUMERICAL EXAMPLES}

To discuss the advantages offered by the present formulation, some numerical examples are presented in this section. All the following examples deal with two dimensional problems with generalized plane strain conditions. For each application, the domain of interest $\Omega$ is meshed with triangular elements. Each node possesses three degrees of freedom: two for the displacement field $\boldsymbol{u}$ and one for the damage field $d$. Furthermore, following the recommendations of Reference 3, the element size $h$ is smaller than $l_{\mathrm{c}} / 4$ in the crack propagation zone.

\section{1 | Notched specimen under tension}

A notched specimen, whose geometry is shown in Figure 1, is considered for the first application of the proposed model. The specimen is meshed with 19300 triangular elements. The typical size $h$ of an element is about $1.5 \times 10^{-3} \mathrm{~mm}$ in the crack propagation zone and $6 \times 10^{-3} \mathrm{~mm}$ elsewhere. A vertical displacement $u$ of $8 \times 10^{-3} \mathrm{~mm}$ is progressively imposed on the top edge of the specimen, whereas the bottom edge is completely fixed. The total duration of the simulation is $10^{-3}$ seconds.

The material properties are listed in Table 1. Stiffness properties, which are assumed to be isotropic, are given by the Young's modulus $E$ and the Poisson's ratio $v$. Also, in order to investigate the impact of the length scale $l_{c}$, this parameter is varied from $0.015 \mathrm{~mm}$ to $0.0075 \mathrm{~mm}$. The resulting crack patterns are displayed in Figure 2 for different vertical displacements. The crack initiates at the notch tip and propagates horizontally. Also, as expected, the sharpest crack pattern is obtained for the smallest length scale parameter $l_{\mathrm{c}}=0.0075 \mathrm{~mm}$. The corresponding load-displacement curves are shown in Figure 3. Because damage development is controlled by $g_{c}$, the load-displacement curve does not depend much on the internal length scale. 


\begin{tabular}{ll} 
Property & Value \\
\hline$E$ & $210 \mathrm{GPa}$ \\
$v$ & 0.3 \\
\hline$g_{\mathrm{c}}$ & $2.7 \times 10^{-3} \mathrm{kN} / \mathrm{mm}$ \\
\hline$l_{\mathrm{c}}$ & $1.5 \times 10^{-2}$ to $7.5 \times 10^{-2} \mathrm{~mm}$ \\
\hline$K$ & $10^{-7} \mathrm{kNs} / \mathrm{mm}^{2}$ \\
\hline$f_{\mathrm{d}}$ & 1 \\
\hline$f_{\mathrm{s}}$ & 1 \\
\hline$N$ & 1 \\
\hline
\end{tabular}

T A B L E 1 Single-edge-notched tensile test: material parameters

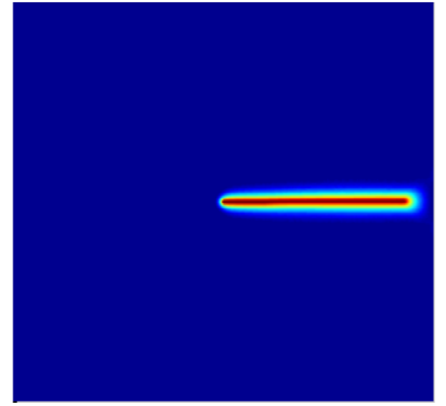

(A)

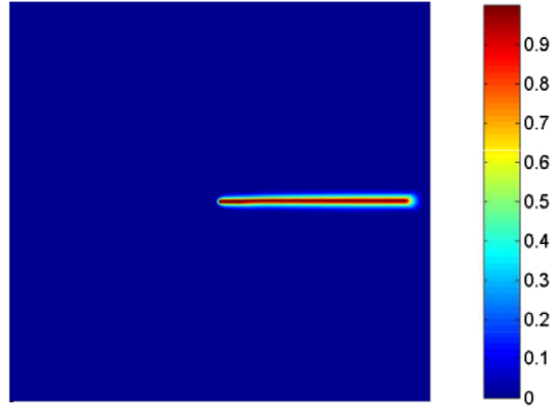

(B)

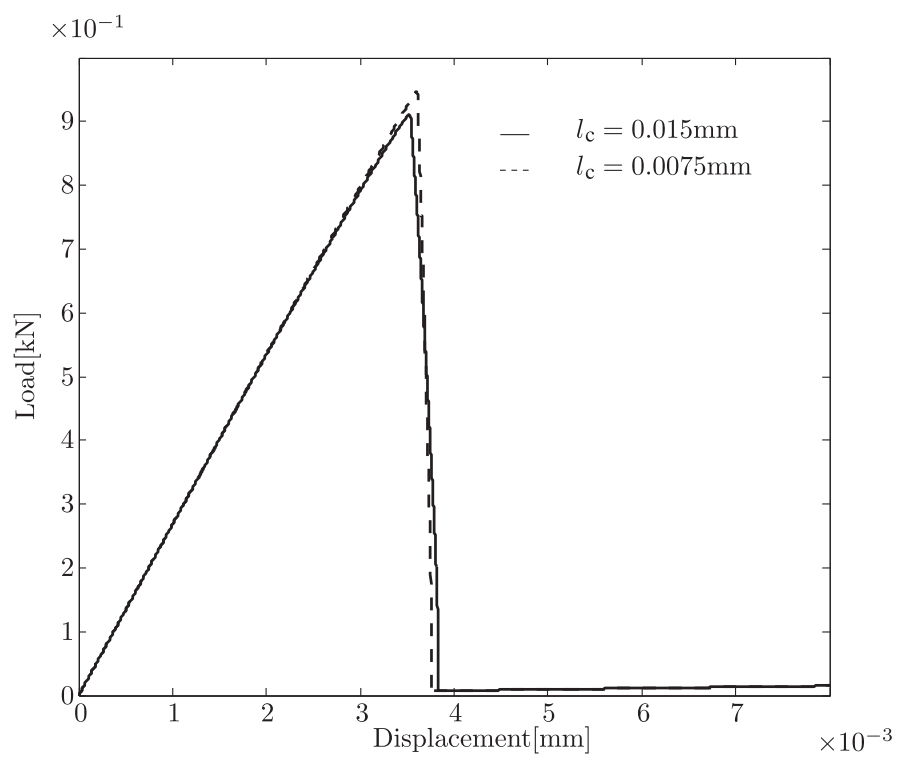

F I G U R E 3 Single-edge-notched tensile test: load-displacement curve for a length scale of $l_{0}=0.015 \mathrm{~mm}$ represented by the solid line and $l_{\mathrm{c}}=0.0075 \mathrm{~mm}$ represented by the dashed line

F I G U R E 2 Single-edge-notched tensile test: crack patterns at a vertical displacement of (A) $3.8 \times 10^{-3} \mathrm{~mm}$ for a length scale $l_{\mathrm{c}}=0.015 \mathrm{~mm}$ and (B) $4 \times 10^{-3} \mathrm{~mm}$ for a length scale $l_{\mathrm{c}}=0.0075 \mathrm{~mm}$ [Colour figure can be viewed at wileyonlinelibrary.com]

\section{2 | Notched specimen under shear}

For the second example, the specimen geometry is the same as before but different boundary conditions are used (see Figure 4). Indeed, following the suggestion of Bourdin et al, ${ }^{28}$ a horizontal displacement of $+u$ (respectively $-u$ ) is progressively imposed on the upper (respectively lower) boundary of the specimen so that the notched specimen is sheared. The total duration of the shear test is $10^{-3}$ seconds and the maximum horizontal displacement is $2 \times 10^{-2} \mathrm{~mm}$. The specimen is meshed with 75500 triangular elements. In order to capture the crack pattern properly, the typical size $h$ of an element is about $10^{-3} \mathrm{~mm}$ in the crack propagation zone and $4 \times 10^{-3} \mathrm{~mm}$ elsewhere. The material properties are presented in Table 2. For this application, the $f_{\mathrm{d}}$ and $f_{\mathrm{s}}$ parameters are varied from 0.1 to 10 . 
F I G U R E 4 Single-edge-notched shear test: boundary conditions, specimen geometry, and mesh [Colour figure can be viewed at wileyonlinelibrary.com]
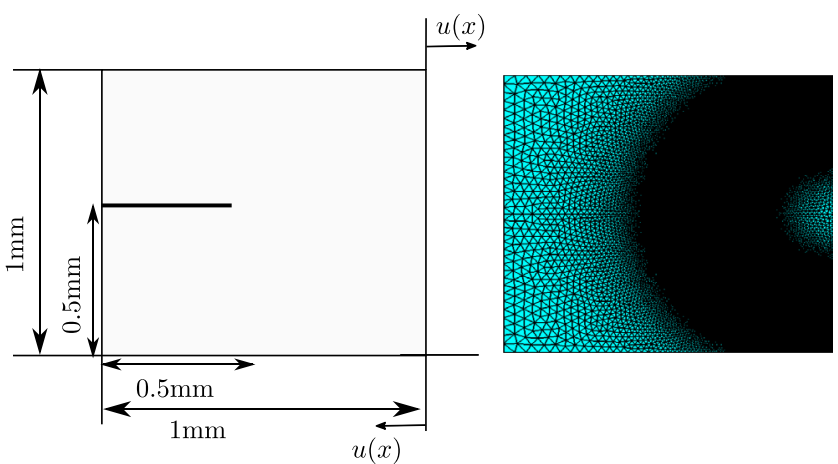

TA B L E 2 Single-edge-notched shear test: material parameters

\begin{tabular}{ll} 
Property & Value \\
\hline$E$ & $210 \mathrm{GPa}$ \\
$v$ & 0.3 \\
\hline$g_{\mathrm{c}}$ & $2.7 \times 10^{-3} \mathrm{kN} / \mathrm{mm}$ \\
\hline$l_{\mathrm{c}}$ & $1.5 \times 10^{-2} \mathrm{~mm}$ \\
\hline$K$ & $10^{-4} \mathrm{kNs} / \mathrm{mm}^{2}$ \\
\hline$f_{\mathrm{d}}$ & from 0.1 to 10 \\
\hline$f_{\mathrm{s}}$ & from 0.1 to 10 \\
\hline$N$ & 1 \\
\hline
\end{tabular}

F I G U R E 5 The role of the $f_{\mathrm{d}}$ and $f_{\mathrm{s}}$ parameters, which can be adjusted to control the tension-compression asymmetry. Single-edge-notched shear test: intermediate patterns for different values of $f_{\mathrm{s}}$ and $f_{\mathrm{d}}$ parameters for a imposed displacement of (A) $u=8 \times 10^{-3} \mathrm{~mm}$, (B) $u=1 \times 10^{-2} \mathrm{~mm}$, and $(\mathrm{C}) u=1.5 \times 10^{-2} \mathrm{~mm}$ [Colour figure can be viewed at wileyonlinelibrary.com]
(A)

(B)

(C)

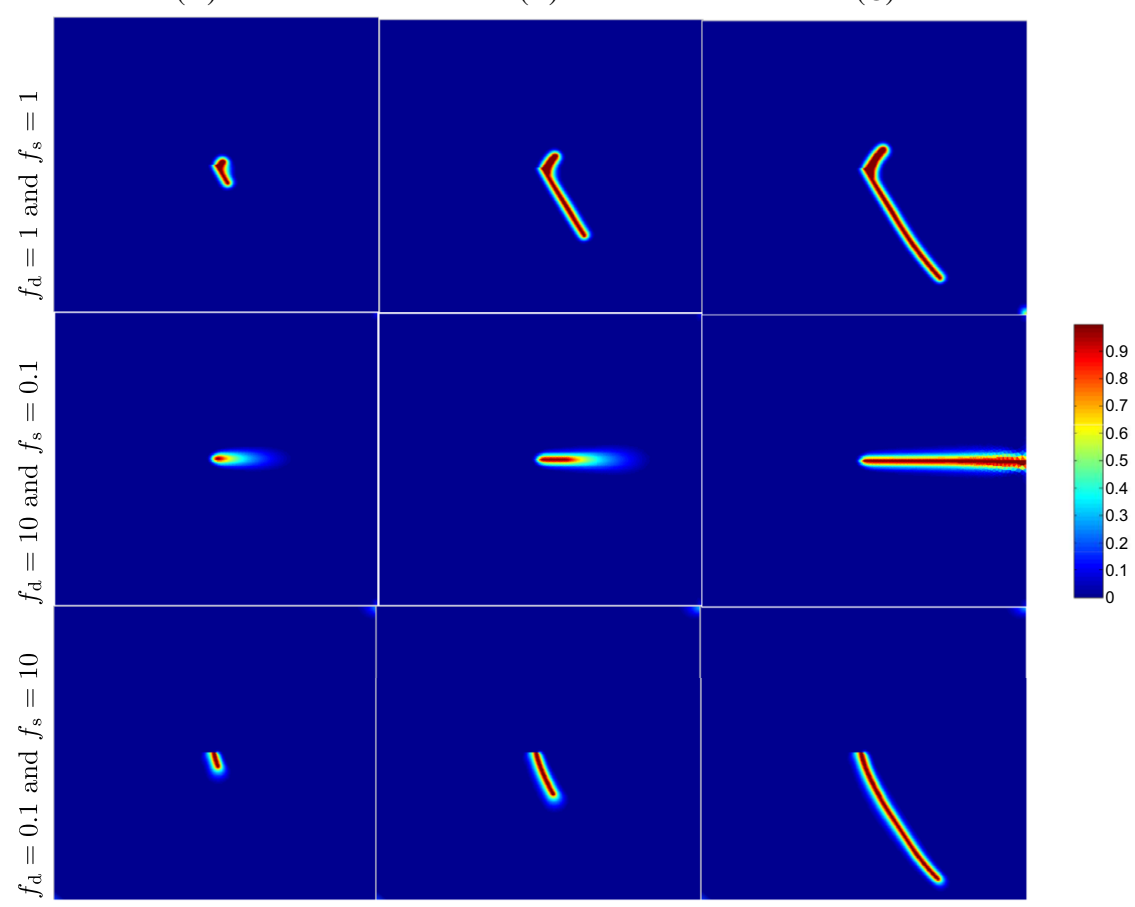

The intermediate crack patterns obtained for the different $f_{\mathrm{d}}$ and $f_{\mathrm{s}}$ values are shown in Figure 5 . According to the results, the crack propagation path can be controlled with the $f_{\mathrm{s}}$ and $f_{\mathrm{d}}$ parameters. Specifically, the crack is forced to propagate downward when the $f_{\mathrm{s}} / f_{\mathrm{d}}$ ratio increases, in which case damage preferably develops in the tensile region of the notched specimen. At the opposite, when $f_{\mathrm{s}} / f_{\mathrm{d}}$ is small, the spherical contribution to the stiffness tensor is not much affected by damage and an horizontal crack propagation is observed. 


\begin{tabular}{llllll}
$r_{1}(\mathrm{~mm})$ & $r_{2}(\mathrm{~mm})$ & $r_{3}(\mathrm{~mm})$ & $h_{1}(\mathrm{~mm})$ & $h_{2}(\mathrm{~mm})$ & $h_{3}(\mathrm{~mm})$ \\
0.4 & 0.41 & 0.8 & 0.4 & 0.8 & 0.5 \\
\hline
\end{tabular}

TA B L E 3 Dimensions of the hat-shaped specimen
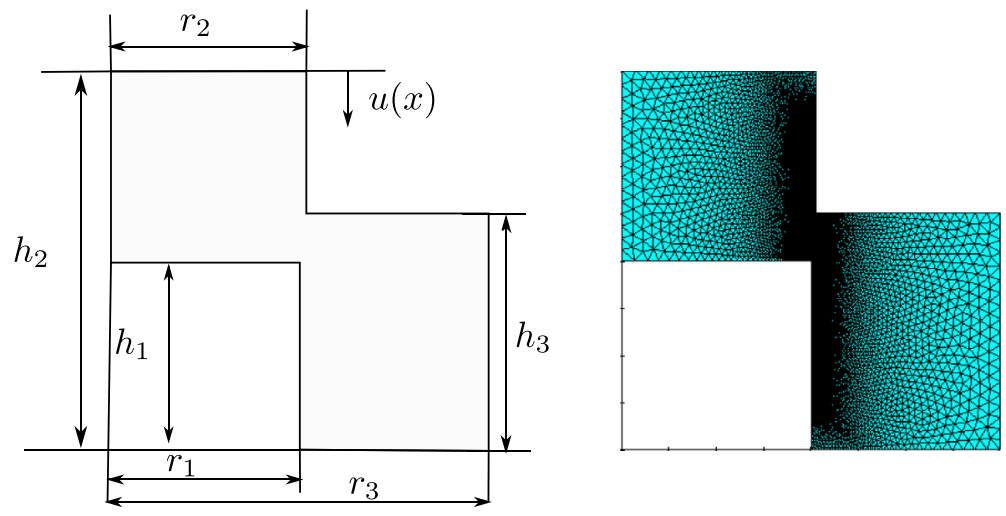

F I G U R E 6 Hat-shaped specimen: boundary conditions, specimen geometry, and mesh [Colour figure can be viewed at wileyonlinelibrary.com]

\begin{tabular}{|ll}
\hline Property & Value \\
\hline$E$ & $210 \mathrm{GPa}$ \\
\hline$v$ & 0.3 \\
\hline$g_{\mathrm{c}}$ & $2.7 \times 10^{-3} \mathrm{kN} / \mathrm{mm}$ \\
\hline$l_{\mathrm{c}}$ & $0.01 \mathrm{~mm}$ \\
\hline$K$ & $10^{-7} \mathrm{kNs} / \mathrm{mm}^{2}$ \\
\hline$N$ & 1 \\
\hline$f_{\mathrm{d}}$ & 0.1 \\
\hline$f_{\mathrm{s}}$ & 10 \\
\hline
\end{tabular}

T A B L E 4 Hat-shaped specimen: material parameters

\section{3 | Hat-shaped specimen}

A hat-shaped specimen with the dimensions given in Table 3 (see Reference 29) is now considered. The top edge is restrained horizontally and displaced vertically up to $6 \times 10^{-3} \mathrm{~mm}$, whereas the bottom edge is totally fixed as shown in Figure 6. The total duration of the test is 1 second. The material parameters are listed in Table 4 . The spatial discretization of the hat-shaped specimen uses 22091 triangular elements, with a local refinement in the central crack propagation zone. For comparison purposes, this problem is investigated with both the proposed model and the model of Reference 4, which relies on the decomposition of the strain tensor into positive and negative contributions.

The damage fields obtained at the end of the simulation for both models are presented in Figure 7. With both models, the development of damage is localized in the central sheared zone. The load vs displacement curves are plotted in Figure 8. With the present model, as one would expect, the applied force is observed to vanish when the specimen is fully broken. At the opposite, according to the model of Reference 4, load transfer through the broken specimen remains possible, which results in a nonvanishing of the applied force. This emphasizes the interest of a deviatoric/spherical decomposition, rather than positive/negative, of the strain tensor to consider closure effects. However, it should be mentioned that, no matter what decomposition method is retained, the stiffness perpendicular to the crack plane is not fully recovered when the crack is closed (ie, when the normal stress acting on the crack plane is negative). This is due to the fact that these models use a scalar damage variable which, by definition, does not provide any information regarding the local orientation of cracks. To circumvent this issue, one must either use a tensorial damage variable ${ }^{30}$ or includes some information regarding the local orientation of cracks with respect to the stress state..$^{20}$ 
F I G U R E 7 Hat-shaped specimen: crack patterns obtained with the present model (A) and the model of Miehe (B) [Colour figure can be viewed at wileyonlinelibrary.com]

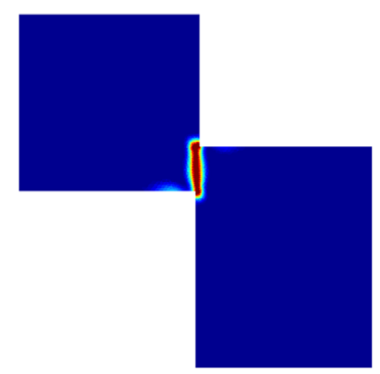

(A)

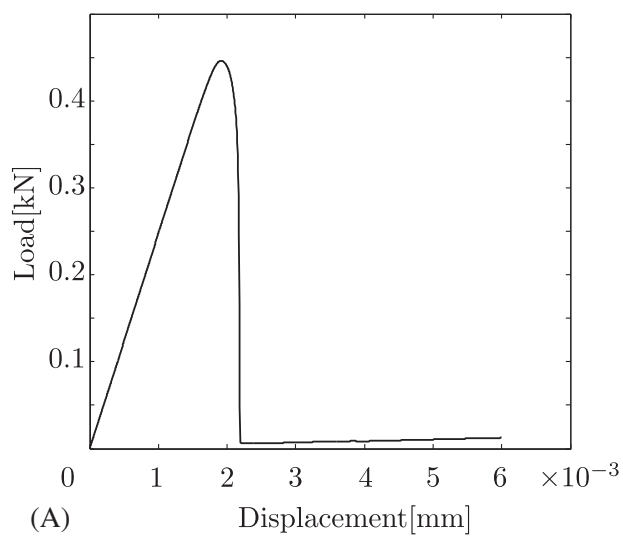

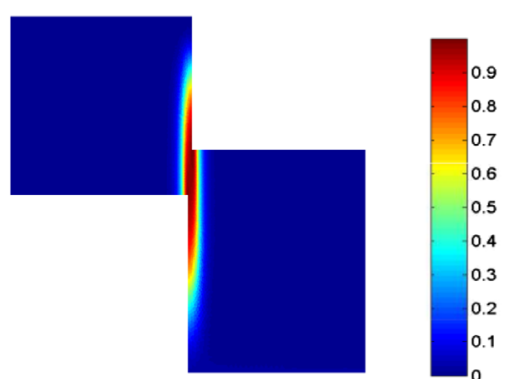

(B)

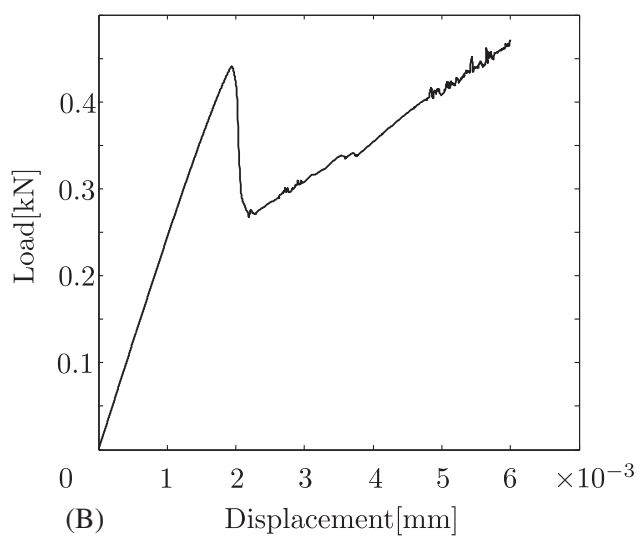

F I G U R E 8 Hat-shaped specimen: load-displacement curve with the present model (A) and the model of Miehe (B)

F I G U R E 9 Polycrystalline microstructure generated from a Voronoi tessellation of 60 randomly distributed seed points. Each color represents a random crystallographic orientation. The dashed-white line represents the hole situated in the middle of the plate [Colour figure can be viewed at wileyonlinelibrary.com]
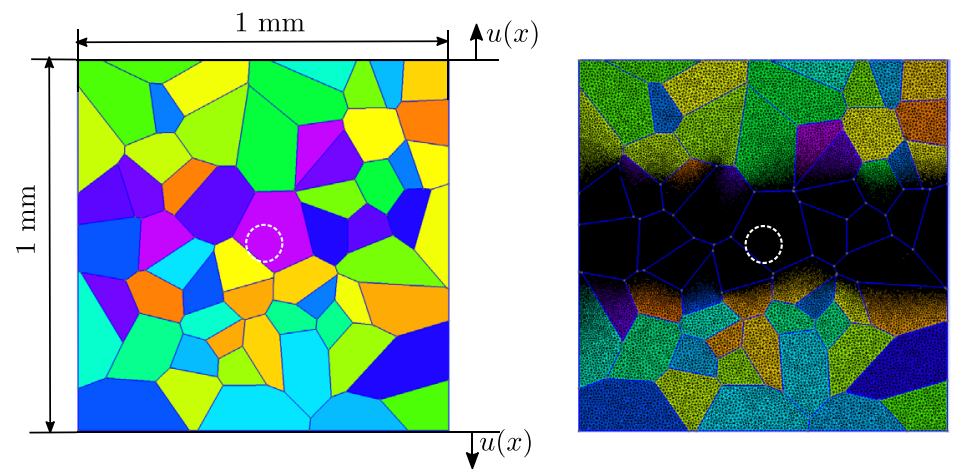

\section{4 | Polycrystalline volume element under tension}

In this section, for illustration purposes, the development of damage in a polycrystalline volume element under plane strain conditions is investigated. As shown in Figure 9, the volume element is a square plate of length $1 \mathrm{~mm}$ containing a central circular predamaged zone (ie, $d=1$ ) of radius $r=0.04 \mathrm{~mm}$. The polycrystalline microstructure has been generated with a Voronoi tessellation of 60 seed points. The crystallographic orientations of the individual grains were assigned randomly. Here, only the specific case of cubic symmetry is considered. In this case, the initial fourth-rank stiffness tensor $\tilde{\mathbb{C}}$ is defined by three independent constants $C_{11}, C_{12}$, and $C_{44}$. Using Voigt notation for two dimensions, such a tensor can be written as:

$$
[\tilde{\mathbb{C}}]=\left(\begin{array}{ccc}
C_{11} & C_{12} & 0 \\
C_{12} & C_{11} & 0 \\
0 & 0 & C_{44}
\end{array}\right) .
$$

For the application of the finite element method, the volume element is meshed with 210000 triangular elements (see Figure 9). The typical size $h$ of an element is about $2 \times 10^{-3} \mathrm{~mm}$ in the crack propagation zone and $10^{-2} \mathrm{~mm}$ in the rest of the domain. 


\begin{tabular}{|c|c|c|c|c|}
\hline \multicolumn{2}{|l|}{ Case } & \multirow{2}{*}{$\begin{array}{l}\mathbf{1} \\
180\end{array}$} & \multirow{2}{*}{$\begin{array}{l}2 \\
180\end{array}$} & \multirow{2}{*}{$\begin{array}{l}3 \\
52\end{array}$} \\
\hline Elasticity & $C_{11}\left(\mathrm{kN} / \mathrm{mm}^{2}\right)$ & & & \\
\hline & $C_{12}\left(\mathrm{kN} / \mathrm{mm}^{2}\right)$ & 104 & 100 & 29 \\
\hline & $C_{44}\left(\mathrm{kN} / \mathrm{mm}^{2}\right)$ & 21 & 40 & 117 \\
\hline & $Z$ & 0.5 & 1 & 10 \\
\hline \multirow[t]{6}{*}{ Damage } & $g_{c}$ & \multicolumn{3}{|c|}{$2.7 \times 10^{-3} \mathrm{kN} / \mathrm{mm}$} \\
\hline & K & \multicolumn{3}{|c|}{$10^{-3} \mathrm{kNs} / \mathrm{mm}^{2}$} \\
\hline & $l_{\mathrm{c}}$ & \multicolumn{3}{|c|}{$10^{-2} \mathrm{~mm}$} \\
\hline & $f_{\mathrm{d}}$ & \multicolumn{3}{|l|}{0.1} \\
\hline & $f_{\mathrm{s}}$ & \multicolumn{3}{|l|}{10} \\
\hline & $N$ & \multicolumn{3}{|l|}{1} \\
\hline
\end{tabular}

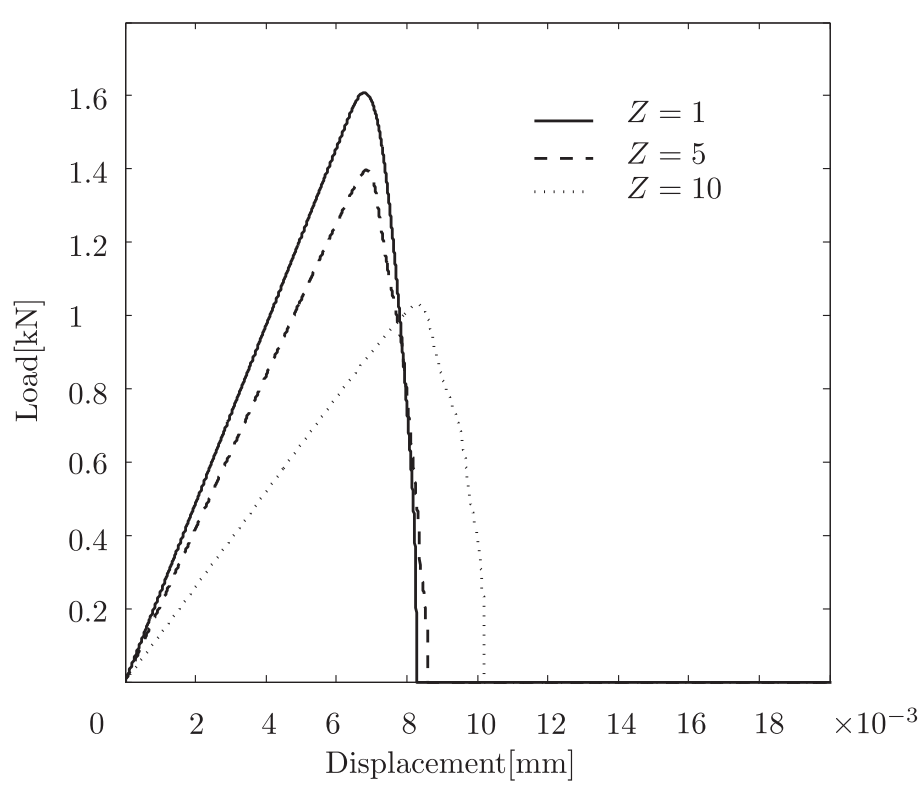

T A B L E 5 Polycrystalline volume element under tension: material parameters for different $Z$ values
F I G U R E 10 Polycrystalline volume element under tension: load-displacement curves for different Zener anisotropy coefficients

The boundary conditions consist of imposing a progressive vertical displacement up to $0.06 \mathrm{~mm}$ to both the top and bottom edges. The duration of the simulation is 1 second.

In order to evaluate the impact of elastic anisotropy on crack propagation, some simulations have been performed with different anisotropy factors. According to Zener, ${ }^{31}$ the anisotropy factor $Z$ for cubic crystals is given by:

$$
Z=2 C_{44} /\left(C_{11}-C_{12}\right) \text {. }
$$

As shown in Table 5, the anisotropy factor $Z$ has been varied from 1 to 10 in the present work. The specific case of isotropy corresponds to $Z=1$. The load-displacement curves are shown in Figure 10 . Whatever the value of $Z$ is, the load completely vanishes when the crack passes through the whole volume element. As illustrated by Figure 11, the crack propagation path is impacted by elastic anisotropy. More specifically, in the isotropic case, the crack follows a straight line as the impact of microstructural heterogeneities is inexistent. At the opposite, when $Z$ is very different from unity, some important deviations along the crack propagation path are observed. For the specific case where $Z$ is equal to 10 , the important internal stresses around a triple junction are responsible for a branching phenomenon.

\section{5 | Notched rectangular plate under dynamic tension}

The case of a polymethyl methacrylate (PMMA) rectangular plate under dynamic loading has been studied experimentally by Zhou. ${ }^{32}$ Several numerical investigations of this problem can also be found in the literature, with different fracture 
F I G U R E 11 Polycrystalline volume element under tension: (A) initial $u=0 \mathrm{~mm}$, (B) $u=7.5 \times 10^{-3} \mathrm{~mm}$, and (C) $u=1.2 \times 10^{-2} \mathrm{~mm}$. The different pictures correspond to different values of the Zener anisotropy coefficient: $Z=1$, $Z=5$, and $Z=10$ [Colour figure can be viewed at wileyonlinelibrary.com]
(A)
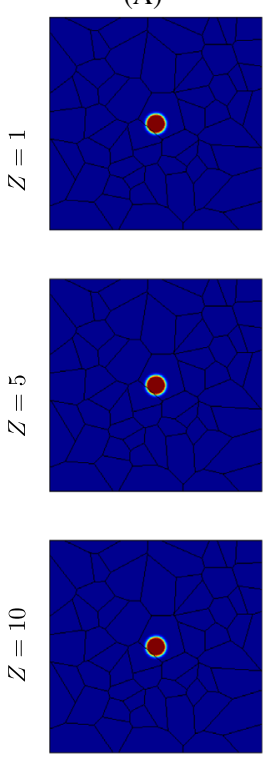

(B)
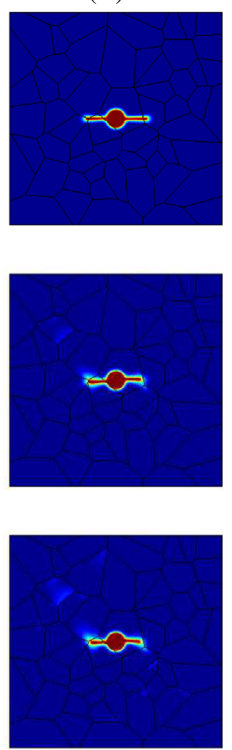

(C)
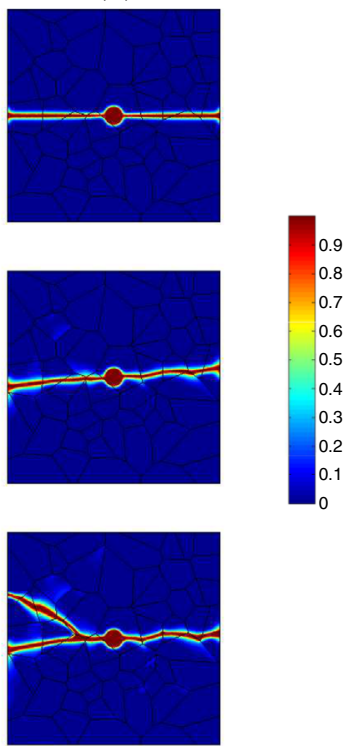

F I G U R E 12 Prestrained PMMA plate problem (left) and experimental results of the specimen broken by branched crack $^{35}$
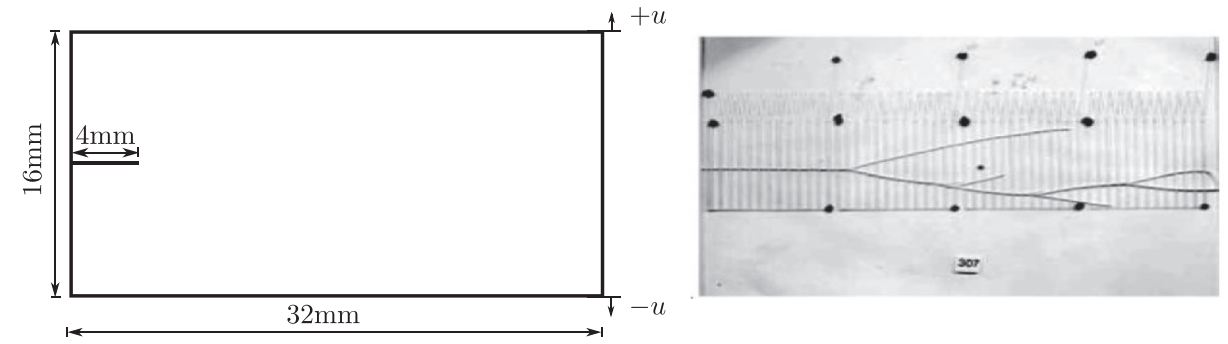

modeling approaches such as cohesive elements, ${ }^{33}$ nonlocal damage modeling, ${ }^{34}$ and phase field modeling. ${ }^{24}$ For this problem, as shown in Figure 12, a crack of length $4 \mathrm{~mm}$ is introduced on the left side of the specimen. The loading conditions are such that the vertical displacement of the upper and lower edges is first progressively increased under quasi-static conditions. Once the maximum displacement is reached, at $t=0$, the explicit dynamic analysis is then started with similar boundary conditions. This implies that there is no energy input to the system during the dynamic analysis. Crack propagation is therefore fed by the elastic energy stored in the plate during the quasi-static analysis.

The evolution of the damage field for different maximum displacements is presented in Figure 13. According to the results, while significant crack branching is observed for $u=0.1 \mathrm{~mm}$ and $u=0.14 \mathrm{~mm}$, no branching phenomenon is detected for the minimum displacement of $0.04 \mathrm{~mm}$.

The evolutions of the total elastic strain energy and total surface energy as a function of time have been plotted in Figure 14. The total elastic strain energy $\mathcal{W}_{\mathrm{e}}$ is given by:

$$
\mathcal{W}_{\mathrm{e}}=\int_{\Omega} \frac{1}{2} \varepsilon: \mathbb{C}: \varepsilon \mathrm{d} V
$$

The total crack surface energy $\mathcal{W}_{\mathrm{s}}$ is evaluated from:

$$
\mathcal{W}_{\mathrm{s}}=\frac{3 g_{\mathrm{c}}}{8 l_{\mathrm{c}}} \int_{\Omega}\left(d+l_{\mathrm{c}}^{2} \nabla d \cdot \nabla d\right) \mathrm{d} V
$$

Because of the development of damage, the elastic strain energy progressively decreases while an augmentation of crack surface energy is observed. Also, when the initial amount of stored elastic strain energy is too excessive for energy to be dissipated with a single crack, the number of crack branches increases, which results in an important augmentation of the surface energy. Branching phenomena are therefore favored for $u=0.14 \mathrm{~mm}$, which corresponds to the case where the initial stored energy quantity is maximal. 

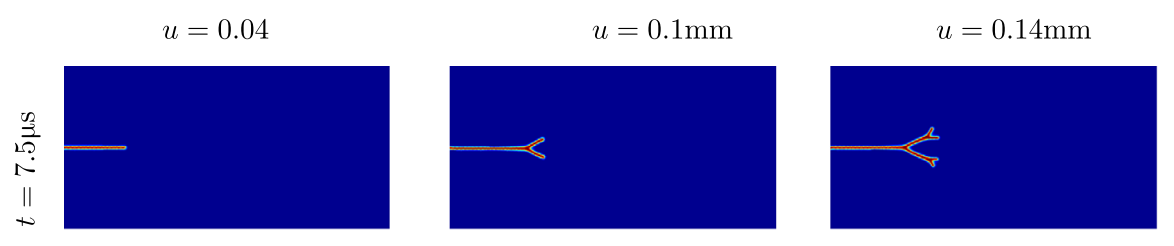

\section{F I G U R E 13 Notched rectangular plate under dynamic tension: damage fields for the different imposed displacements and different times [Colour figure can be viewed at wileyonlinelibrary.com]}
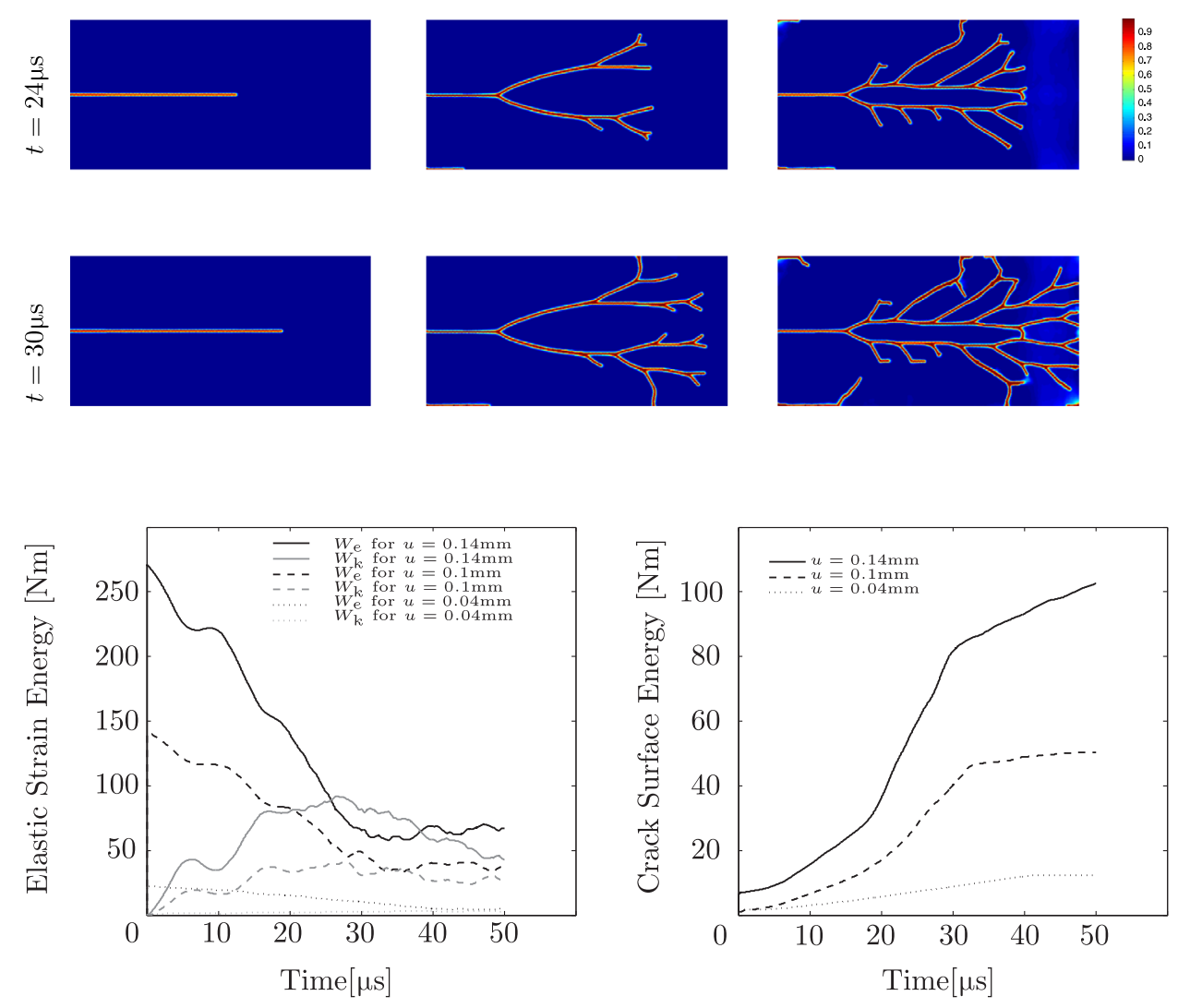

F I G U R E 14 Notched rectangular plate under dynamic tension: Evolutions of the total elastic strain energy $\mathcal{W}_{\mathrm{e}}$ and the kinetic energy $\mathcal{W}_{\mathrm{k}}$ (left) and total surface energy $\mathcal{W}_{\mathrm{s}}$ (right)

For a more quantitative approach, the total crack length $l$ and crack velocity $v$ have been evaluated with the following expressions:

$$
l=\frac{1}{2} \int_{\Omega} \frac{\nabla d \cdot \nabla d}{\|\nabla d\|} \mathrm{d} V=\frac{1}{2} \int_{\Omega}\|\nabla d\| \mathrm{d} V \text { and } v=\frac{\mathrm{d} l}{\mathrm{~d} t}
$$

The evolution of the length $l$, which is a pure geometric estimation of the half of the separation line length (between $d=0$ and $d=1$ ), is plotted in Figure 15A. According to the results, a rise of the imposed displacement causes an increase in the number of microcracks, hence in the total crack length. This effect is particularly visible for the case $u=0.14 \mathrm{~mm}$.

The evolution of the crack velocity is reported in Figure 15B. Whatever the values of the different displacements are, important fluctuations of the crack velocity are obtained. Such fluctuations have been previously observed experimentally by Fineberg et $\mathrm{al}^{36}$ and numerically by $\mathrm{Xu}$ and Needleman. ${ }^{37}$ These fluctuations are caused by branching. Indeed, when branching occurs, the energy flowing into the crack tip is split between the primary crack and the secondary cracks. Less energy is therefore directed into the primary crack, which causes its velocity to decrease. Once secondary cracks, which enter in competition with the primary crack, have stopped propagating, the primary crack starts growing again until the next branching event is observed. The crack velocity drop, which is observed at the end of the test, corresponds to the situation where the crack has propagated through the specimen. 
F I G U R E 15 Notched rectangular plate under dynamic tension: evolutions of the crack length $l$ (left) and the normalized crack velocity $v / c_{R}$ (right). $c_{R}$ is the Rayleigh wave speed, which is about $906 \mathrm{~m} / \mathrm{s}$ for PMMA
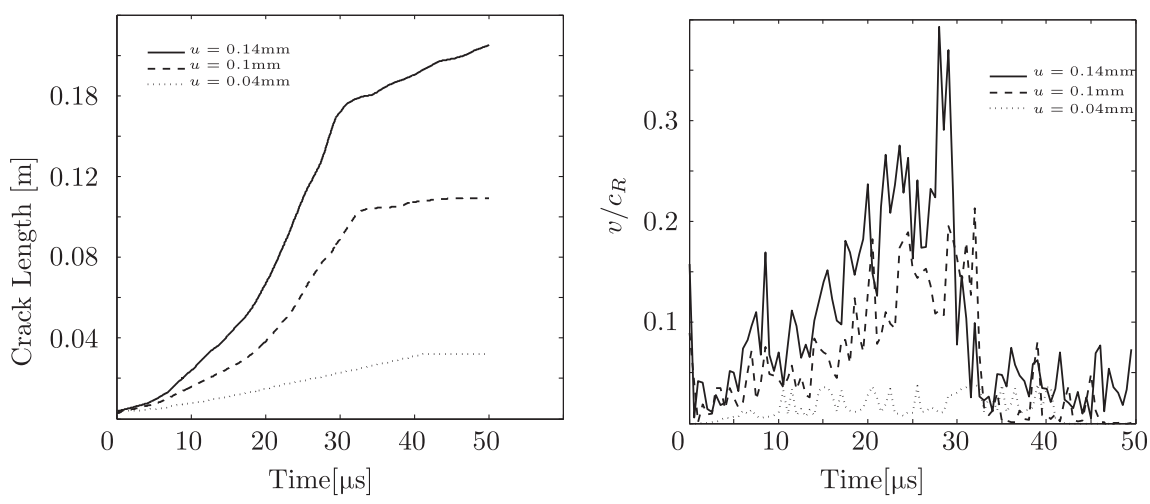

F I G U R E 16 Geometry and boundary conditions for the notched plate under impact [Colour figure can be viewed at wileyonlinelibrary.com]

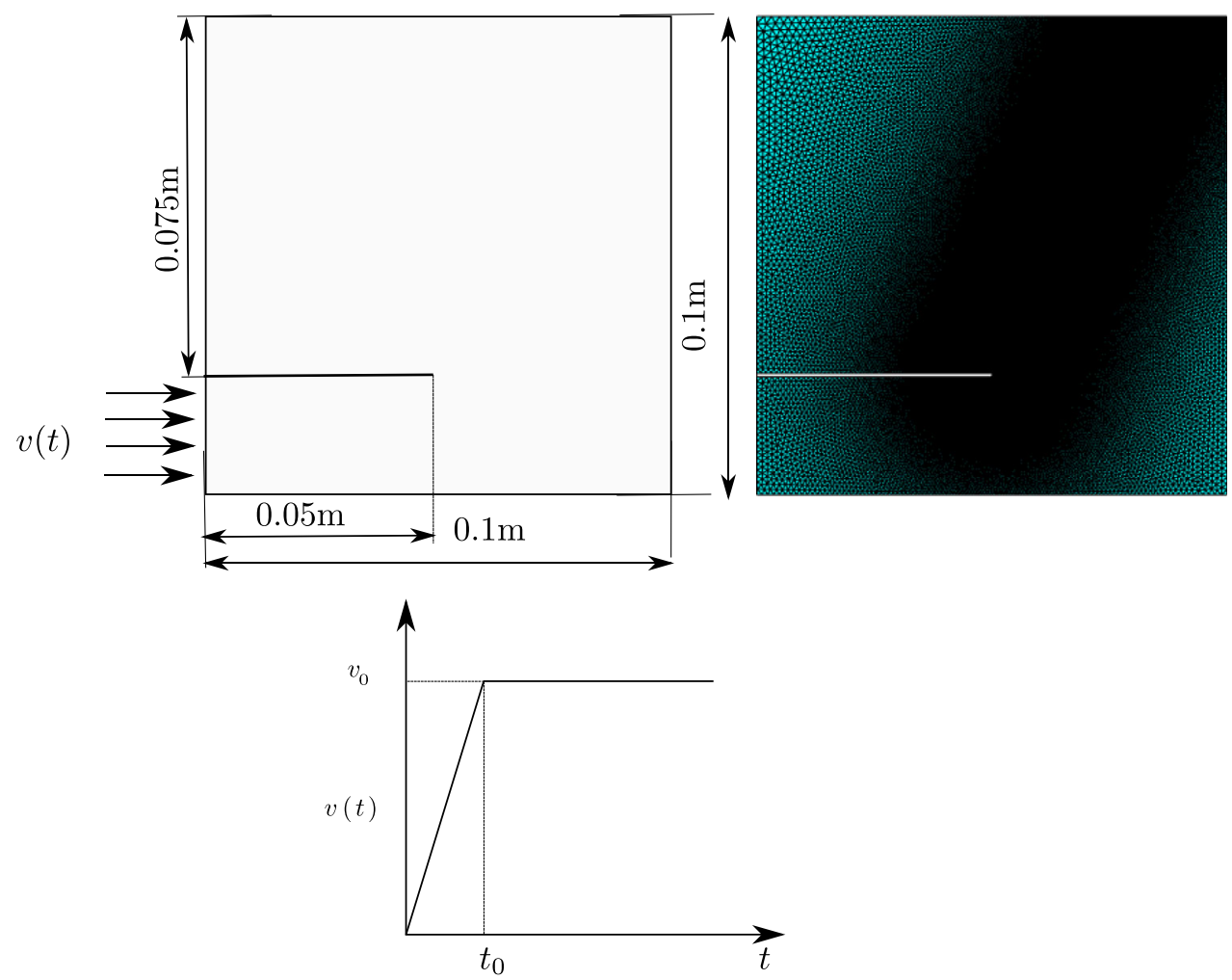

\section{6 | Notched plate under impact}

In this last example, the case of crack propagation under a dynamic shear loading is investigated. The numerical model is based on the experimental results reported by Kalthoff and Winkler ${ }^{38}$ and Kalthoff. ${ }^{39}$ The experiment of Kalthoff and Winkler ${ }^{38}$ consists in impacting a prenotched plate with a projectile. This example has been studied by Song et $\mathrm{al}^{40}$ and by Remmers et $\mathrm{al}^{41}$ with cohesive elements and with phase field modeling by Borden et al ${ }^{15}$ and Hofacker et al. ${ }^{5}$

In the present case, due to symmetry considerations, only the upper half part of the plate is considered to reduce the computational cost. The geometry and boundary conditions for the reduced problem are described in Figure 16. The plate is meshed with 89120 triangular elements with a refined mesh in areas where the crack is expected to propagate. The impact velocity $v$ is applied on the bottom part of the left edge. The imposed velocity $v$ is first increased from zero to $v_{0}$ during a transient period of $t_{0}=10^{-7}$ seconds. It is then held constant during the remaining part of the test. The material properties used for this numerical test are listed in Table 7.

The damage fields, which have been obtained for two different impact velocities $v_{0}=40 \mathrm{~m} / \mathrm{s}$ and $v_{0}=70 \mathrm{~m} / \mathrm{s}$, are presented in Figure 17. According to the results, whatever the impact velocity is, the crack propagation direction is consistent with the experimental findings of Kalthoff, ${ }^{39}$ who observed cracks to propagate with an angle of about $70^{\circ}$ with respect to the horizontal direction. Also, when the impact velocity is increased to $70 \mathrm{~m} / \mathrm{s}$, a branching phenomenon occurs. 


\begin{tabular}{|ll}
\hline Property & Value \\
\hline$E$ & $3.09 \mathrm{GPa}$ \\
\hline$v$ & 0.35 \\
\hline$c_{\mathrm{R}}$ & $906 \mathrm{~m} / \mathrm{s}$ \\
\hline$g_{\mathrm{c}}$ & $300 \mathrm{~J} / \mathrm{m}^{2}$ \\
\hline$l_{\mathrm{c}}$ & $0.1 \mathrm{~mm}$ \\
\hline$K$ & $10^{-3} \mathrm{Ns} / \mathrm{m}^{2}$ \\
\hline$\rho$ & $1180 \mathrm{~kg} / \mathrm{m}^{3}$ \\
\hline$N$ & 1 \\
\hline$f_{\mathrm{d}}$ & 0.1 \\
\hline$f_{\mathrm{s}}$ & 10 \\
\hline
\end{tabular}

TA B L E 6 Notched rectangular plate under dynamic tension: material parameters

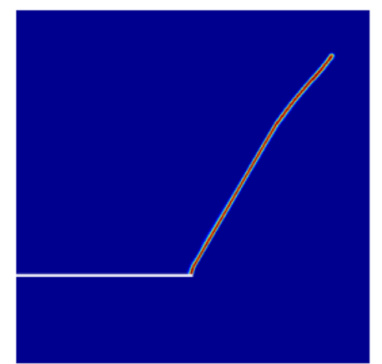

(A)

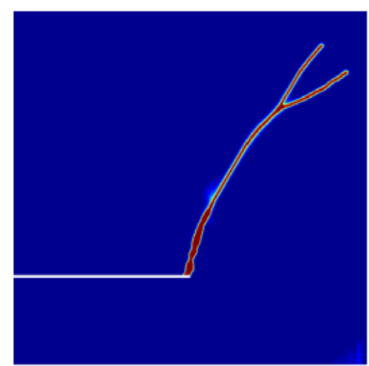

(B)
F I G U R E 17 Notched plate under impact: damage crack patterns for the imposed velocities of $(\mathrm{A}) v_{0}=40 \mathrm{~m} / \mathrm{s}$ and $(\mathrm{B}) v_{0}=70 \mathrm{~m} / \mathrm{s}$ [Colour figure can be viewed at wileyonlinelibrary.com]

\begin{tabular}{ll} 
Property & Value \\
\hline$E$ & $190 \mathrm{GPa}$ \\
$v$ & 0.3 \\
\hline$c_{\mathrm{R}}$ & $2803 \mathrm{~m} / \mathrm{s}$ \\
\hline$g_{\mathrm{c}}$ & $2.217 \times 10^{4} \mathrm{~J} / \mathrm{m}^{2}$ \\
\hline$l_{\mathrm{c}}$ & $0.3 \mathrm{~mm}$ \\
\hline$K$ & $10^{-3} \mathrm{Ns} / \mathrm{m}^{2}$ \\
\hline$\rho$ & $8000 \mathrm{~kg} / \mathrm{m}^{3}$ \\
\hline$N$ & 1 \\
\hline$f_{\mathrm{d}}$ & 0.1 \\
\hline$f_{\mathrm{s}}$ & 10 \\
\hline
\end{tabular}

T A B L E 7 Notched rectangular plate under dynamic shear loading: material parameters

\section{7 | CONCLUSION}

In this work, a phase field implementation of a new damage gradient model for brittle fracture is developed to address the simulation of crack nucleation and propagation in anisotropic materials. The model uses a scalar damage variable to represent the progressive degradation of mechanical resistance. The spatial gradient of the damage variable, which is treated as an additional external state variable, serves regularization purposes and allows considering the surface energy associated with cracks. Constitutive relations are developed within the framework of generalized standard materials. The coupling of damage with elasticity is considered. Also, the impact of the loading mode on the development of damage is captured by differentiating the influence of spherical and deviatoric parts of the stiffness tensor and considering closure effects. The proposed formulation satisfies the continuity of the stress-strain relation and is adapted to any class of 
material symmetry. Numerical implementation is undertaken via the finite element method, where nodal degrees of freedom are the displacement and the damage variable. According to the numerical examples, the proposed model allows capturing some important aspects of crack propagation, including crack branching and bifurcation. Also, when total failure occurs, the load displacement curves show a completely vanishing force. The main advantage of this method is its ability to produce complex crack patterns, including branching and merging, in both two and three dimensions. It should be noted that the PFM may suffer from the high computational cost (sufficiently refined mesh in the damaged zone is necessary to accurately resolve the gradient term). Even so, this problem can be solved using parallel implementations and adaptive remeshing. Therefore, the way in which the parameters are identified is quite complicated. Moreover, the powerful character of Equation (49) imposes an implicit integration. In the future, a similar strategy will be employed to deal with more complex deformation mechanisms. More specifically, future work will focus on the construction of a model, which includes the possible coupling of damage with plasticity and hardening.

\section{ORCID}

Hela Gmati (10) https://orcid.org/0000-0002-9945-5536

Amine Ammar (1) https://orcid.org/0000-0002-1541-1115

\section{REFERENCES}

1. Griffith AA. The phenomena of rupture and flow in solids. Philos Trans R Soc A Math Phys Eng Sci. 1921;221(582-593):163-198.

2. Francfort G, Marigo J-J. Revisiting brittle fracture as an energy minimization problem. J Mech Phys Solids. 1998;46(8):1319-1342.

3. Miehe C, Welschinger F, Hofacker M. Thermodynamically consistent phase-field models of fracture: variational principles and multi-field Fe implementations. Int J Numer Methods Eng. 2010;83(10):1273-1311.

4. Miehe C, Hofacker M, Welschinger F. A phase field model for rate-independent crack propagation: robust algorithmic implementation based on operator splits. Comput Methods Appl Mech Eng. 2010;199(45):2765-2778.

5. Hofacker M, Miehe C. A phase field model of dynamic fracture: robust field updates for the analysis of complex crack patterns. Int J Numer Methods Eng. 2013;93(3):276-301.

6. Lemaitre PJ. A Course on Damage Mechanics. 2nd ed. Berlin, Heidelberg: Springer-Verlag; 1996.

7. Sicsic P, Marigo J-J. From gradient damage laws to Griffith's theory of crack propagation. J Elast. 2013;113(1):55-74.

8. Nguyen Q-S. Some remarks on standard gradient models and gradient plasticity. Math Mech Solid. 2015;20(6):760-769.

9. Linder C, Armero F. Finite elements with embedded branching. Finite Elem Anal Des. 2009;45(4):280-293. (The Twentieth Annual Robert J. Melosh Competition).

10. Moës N, Dolbow J, Belytschko T. A finite element method for crack growth without remeshing. Int J Numer Methods Eng. 1999;46(1):131-150.

11. Moës N, Stolz C, Bernard PE, Chevaugeon N. A level set based model for damage growth: the thick level set approach. Int J Numer Methods Eng. 2011;86(3):358-380.

12. Nguyen T, Yvonnet J, Zhu Q-Z, Bornert M, Chateau C. A phase field method to simulate crack nucleation and propagation in strongly heterogeneous materials from direct imaging of their microstructure. Eng Fract Mech. 2015;139:18-39.

13. Larsen CJ. Models for Dynamic Fracture Based on Griffith's Criterion. Dordrecht, The Netherlands: Springer; 2010:131-140.

14. Bourdin B, Larsen CJ, Richardson CL. A time-discrete model for dynamic fracture based on crack regularization. Int J Fract. 2011;168(2):133-143.

15. Borden MJ, Verhoosel CV, Scott MA, Hughes TJ, Landis CM. A phase-field description of dynamic brittle fracture. Comput Methods Appl Mech Eng. 2012;217-220:77-95.

16. Hakim V, Karma A. Crack path prediction in anisotropic brittle materials. Phys Rev Lett. 2005;95:235501.

17. Teichtmeister S, Kienle D, Aldakheel F, Keip M-A. Phase field modeling of fracture in anisotropic brittle solids. Int J NonLinear Mech. 2017;97:1-21.

18. Amor H, Marigo J-J, Maurini C. Regularized formulation of the variational brittle fracture with unilateral contact: numerical experiments. J Mech Phys Solids. 2009;57(8):1209-1229.

19. Ambati M, Gerasimov T, De Lorenzis L. A review on phase-field models of brittle fracture and a new fast hybrid formulation. Comput Mech. 2015;55(2):383-405.

20. Steinke C, Kaliske M. A phase-field crack model based on directional stress decomposition. Comput Mech. 2019;63(5):1019-1046.

21. Bourdin B, Francfort G, Marigo J-J. The variational approach to fracture. J Elast. 2008;91(1-3):5-148.

22. Pham K, Amor H, Marigo J-J, Maurini C. Gradient damage models and their use to approximate brittle fracture. Int J Damage Mech. 2011;20(4):618-652.

23. Borden MJ, Hughes TJ, Landis CM, Verhoosel CV. A higher-order phase-field model for brittle fracture: formulation and analysis within the isogeometric analysis framework. Comput Methods Appl Mech Eng. 2014;273:100-118.

24. Bleyer J, Roux-Langlois C, Molinari J-F. Dynamic crack propagation with a variational phase-field model: limiting speed, crack branching and velocity-toughening mechanisms. Int J Fract. 2017;204(1):79-100.

25. Frémond M, Nedjar B. Damage, gradient of damage and principle of virtual power. Int J Solids Struct. 1996;33(8):1083-1103. 
26. Polizzotto C, Borino G. A thermodynamics-based formulation of gradient-dependent plasticity. Eur J Mech A Solid. 1998;17(5):741-761.

27. Halphen B, Son NQ. Sur les materiaux standards generalises [On generalized standard materials]. J Mech. 1975;14(1):39-63. cited by 684.

28. Bourdin B, Francfort G, Marigo J-J. Numerical experiments in revisited brittle fracture. J Mech Phys Solids. 2000;48(4):797-826.

29. Bronkhorst C, Cerreta E, Xue Q, Maudlin P, Mason T, Gray G. An experimental and numerical study of the localization behavior of tantalum and stainless steel. Int J Plast. 2006;22(7):1304-1335.

30. Lubarda V, Krajcinovic D. Damage tensors and the crack density distribution. Int J Solids Struct. 1993;30(20):2859-2877.

31. Zener CM, Siegel S. Elasticity and anelasticity of metals. J Phys Colloid Chem. 1949;53(9):1468-1468.

32. Zhou F. Study on the macroscopic behavior and the microscopic process of dynamic crack propagation [PhD thesis]. University of Tokyo; 1996.

33. Zhou F, Molinari J-F, Shioya T. A rate-dependent cohesive model for simulating dynamic crack propagation in brittle materials. Eng Fract Mech. 2005;72(9):1383-1410.

34. Wolff C, Richart N, Molinari J-F. A non-local continuum damage approach to model dynamic crack branching. Int J Numer Methods Eng. 2015;101(12):933-949.

35. SH. On the crack propagation in brittle materials [PhD thesis]. Japan: Department of Aeronautics and Astronautic, University of Tokyo; 1996.

36. Fineberg J, Gross SP, Sharon E. Micro-branching as an instability in dynamic fracture. In: Willis JR, ed. IUTAM Symposium on Nonlinear Analysis of Fracture. Dordrecht, The Netherlands: Springer; 1997:177-189.

37. Xu X-P, Needleman A. Numerical simulations of fast crack growth in brittle solids. J Mech Phys Solids. 1994;42(9):1397-1434.

38. Winkler S, Kalthoff JF. Failure mode transition of high rates of shear loading. In: Chiem CY, Kunze HD, Meyer LW, eds. Impact Loading and Dynamic Behaviour of Materials. Oberursel, Germany: Deutsche Gesellschaft für Metallkunde, DGM-Verlag; 1987:185-195.

39. Kalthoff JF. Modes of dynamic shear failure in solids. Int J Fract. 2000;101(1):1-31.

40. Song J-H, Wang H, Belytschko T. A comparative study on finite element methods for dynamic fracture. Comput Mech. 2008;42(2):239-250.

41. Remmers JJC, Borst RD, Needleman A. A cohesive segments method for the simulation of crack growth. Comput Mech. 2003;31(1):69-77.

\section{SUPPORTING INFORMATION}

Additional supporting information may be found online in the Supporting Information section at the end of this article.

How to cite this article: Gmati H, Mareau C, Ammar A, El Arem S. A phase-field model for brittle fracture of anisotropic materials. Int J Numer Methods Eng. 2020;1-20. https://doi.org/10.1002/nme.6361 